\title{
Parametric Design of Outdoor Broadcasting Studio Based on Schema Theory
}

\author{
Li Zhu ${ }^{1}$, Nan Zhang ${ }^{1, a}$ and Xiaoying Qing ${ }^{2}$ \\ ${ }^{1}$ School of Architecture and Art, Central South University, Changsha, China \\ ${ }^{2}$ School of Art and Design, Luoyang Institute of Science and Technology, Luoyang, China
}

\begin{abstract}
This paper mainly demonstrates that the schema is an important way for the architect to cognize architecture form logic. It connects schema to algorithm of parametric design in order to seek the "algorithm schema" generation in parametric design of architecture. Meanwhile, this paper discusses the generative process and methods of the "algorithm schema" in parametric design of architecture by describing a case of outdoor broadcasting studio of Hunan Economic Radio. It also reveals the importance of "algorithm schema" for the cognition and architectural form logic generation.
\end{abstract}

\section{Introduction}

Schema is an abstract structure extracted from cognitive experience which appears repeatedly in human life. It helps cognize the structural cognition among objective matters. The concept "schema" could be dated back to the ancient Greek philosopher Plato's basic thought: the ideal is to abstract things in a general way. Based on this idea, people form their cognitions about the world. The study of schema exerts a far-reaching influence not only in philosophy but also in psychology. F.C.Bartlett, a British psychologist, has officially put forward the concept of schema from the view of psychology.He argued that schema helps actively organize the past experience and affect the well adaptable organic reaction. According to cognitive linguistics: schema contains space structure and movement structure in the development. So it plays an important role in the process of conceptual metonymy.

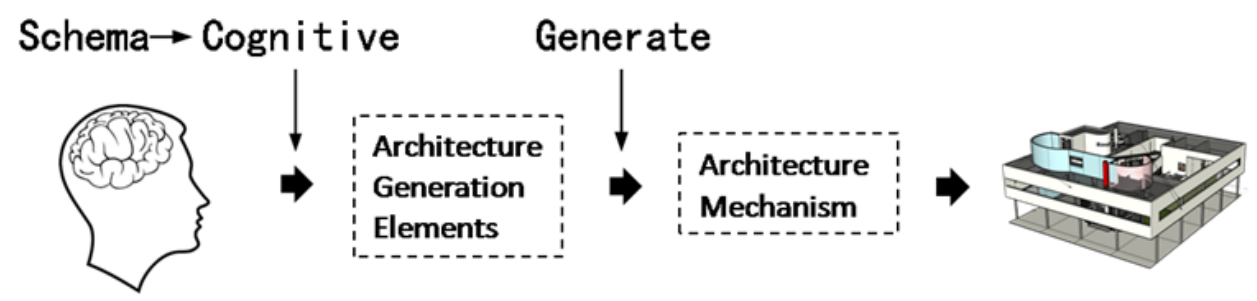

Figure 1. Construction of Architecture Mechanism Based on Schema Theory

\footnotetext{
${ }^{\mathrm{a}}$ Corresponding author : 307579716@qq.com
} 
The analysis above shows that schema can be considered as a very important mean of cognizing the abstract structure of relationship among objects. "Schema is the process of conceiving the structural image based on the conception", said by Immanuel Kant. By this token, the architecture form logic generation also has the same features as schema. Schema could help architects establish the relationship between visual form and elements that affect architecture form generation, such as functional logic of architecture, geographical environment, regional culture, social relations, etc. In this way, schema could help architects cognize the meaning implied in architecture form logic and further improve it.

\section{The schema of parametric architectural design}

The schema conception has been widely used in architectural design. Under the modern science environment which is dominated by Chaos theory, System theory and Control Theory, parametric design is a method that employs the powerful computing capability of computers to describe the architectural form logic. It has renewed the cognition and application of schema in architecture design. The logic of relations among different elements that could affect architecture formation is described by parametric design through an abstract algorithm of computer, thereby generating the non-linear space. Computer algorithm also has the same feature as the schema that could describe the abstract logical relations among different things, therefore we can extract the imaged cognitive schema to algorithm and provide a guide to architecture form generation.

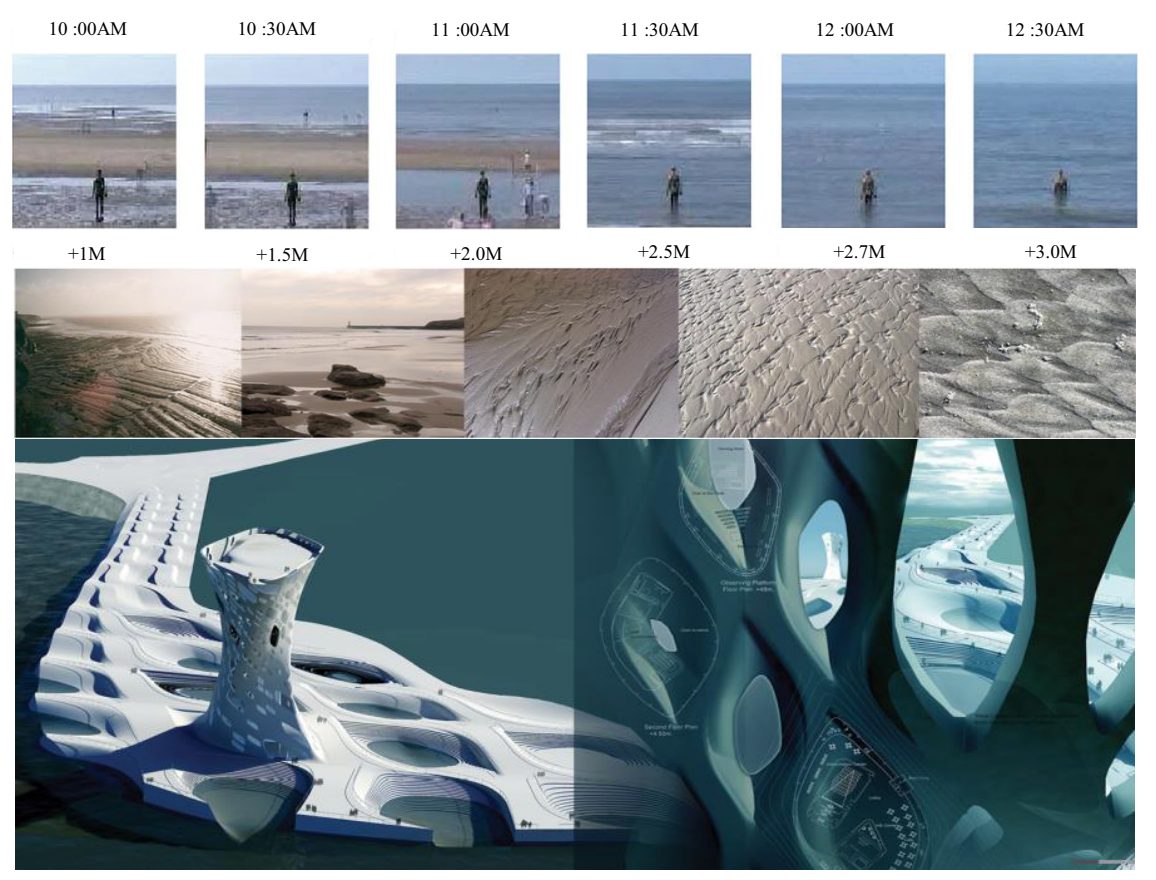

Figure 2. Perceptual schema extraction from the tides

Pure design makes perfectly explanation about the relationship between architecture's form and the logic of tidal action by using computer algorithm on the sightseeing tower project of Mersey river. This project is located at the estuary of Mersey River, with the aim to attract tourists to enjoy the beautiful natural scenery. Pure Architecture expects that the sightseeing tower is not only a place for tourists to enjoy the scenery but also a way to make it possible for tourists to cognize the relationship between architecture's form and the site. Based on the regularity of tidal action of the Mersey River, Pure Architecture designed this special "Tides Park". It is consisted of a series of interweaved paths 
and landscape units. These units include local plants and different heights of water landscape formed by tides. The tower indicates the way how the site was transformed from land into water.

\section{The algorithm schema architecture of a parametric design for outdoor broadcasting studio}

The algorithm schema architecture in parametric design has two levels: firstly, the architect forms the perceptual schema through his cognition and extraction from the generating factors of the architecture. Secondly, architects translate the perceptual schema into an algorithm schema by using computer programming language. As human's abstract cognition runs through the whole process from the extraction of the perceptual schema to the translation of the algorithm schema, the relation among the factors that have effects on architectural form logic converts human's perceptual schema into the algorithm schema, thus forms the logic of architecture generation.

Take the outdoor studio of Hunan Economic Radio as an example, we can explore the relationship between the form logic of the architecture and its algorithm schema. The studio is located at the South Huangxing Roadpedestrian Mall, an important commercial district in Changsha, which is convenient for live broadcast and real-time interaction, besides, this area is full of fashion and modern elements, the architecture form can be easily perceived, it's easy to become a landmark of the city. In general, people perceive the live broadcast in the form of sound through radio channels, however, with the help of outdoor studio, people can not only hear but also see the scene.

\subsection{The schema generation of architecture form logic}

As for the study of the architecture form logic on this case, we will extract the perceptual schema from its functional image. The function of the studio is used for radio program production and broadcast. Here the program is output in the form of wave with different frequency and transmitted to the audience's receiving equipment within its signal coverage and then plays. In this process, the acoustic wave is converted into radio wave first and then transmits in the space, the receiving equipment then catches the wave and restores it back to the acoustic wave, so the audience can perceive the program. The wave becomes the vector that transmits and communicates with the program, so in our formfinding process on this case, since the form of wave has strong functional identification and can be perceived by the viewer in his intuition. The extraction form the perceptual schema of wave becomes starting point of architecture form logic generation.

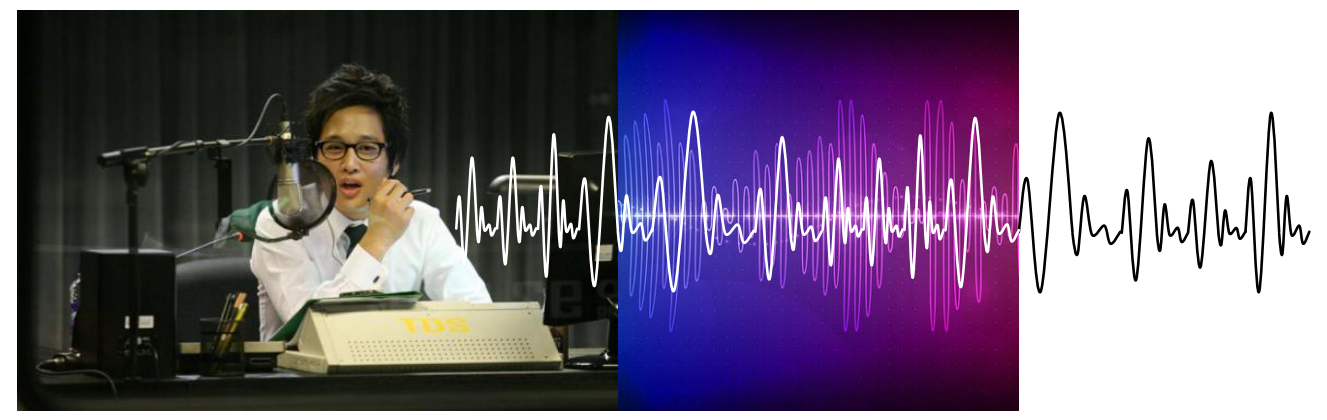

Figure 3. Acoustic wave is converted into radio wave first and then transmits in the space

For this case of the architecture form logic, we select wave image as the perceptual schema to generate the architecture prototype. Parameters such as period, amplitude, are able to affect the twodimensional shape of the wave. After translating the two-dimensional into three-dimensional one, period and amplitude also has the possibility to shape the studio of three-dimensional architecture mass: period controls the depth dimension while amplitude controls the height of the studio and size 
of the bay. Afterwards, by increasing and moving the control point to adjust the modeling of the studio, we can obtain a preliminary architecture.

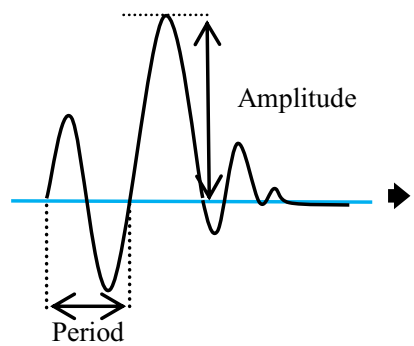

Wave in 2D

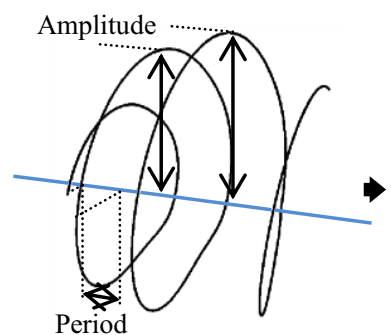

Wave in 3D

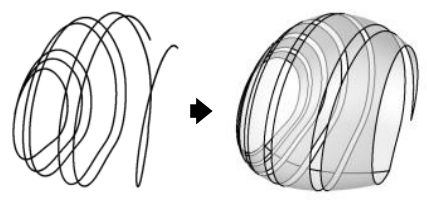

Architecture Prototype

Figure 4. Period and amplitude as parameter of wave control and generate the shape of studio

The original architecture mass is confirmed by two parameters of wave, namely period and amplitude while the frequency affects the form of wave segmenting the evaluation of the architecture. After a comprehensive consideration of the function, form, aesthetic appreciation and mass of the studio, a section of frequency with 6 periods of wave was confirmed to be the prototype of the studio.
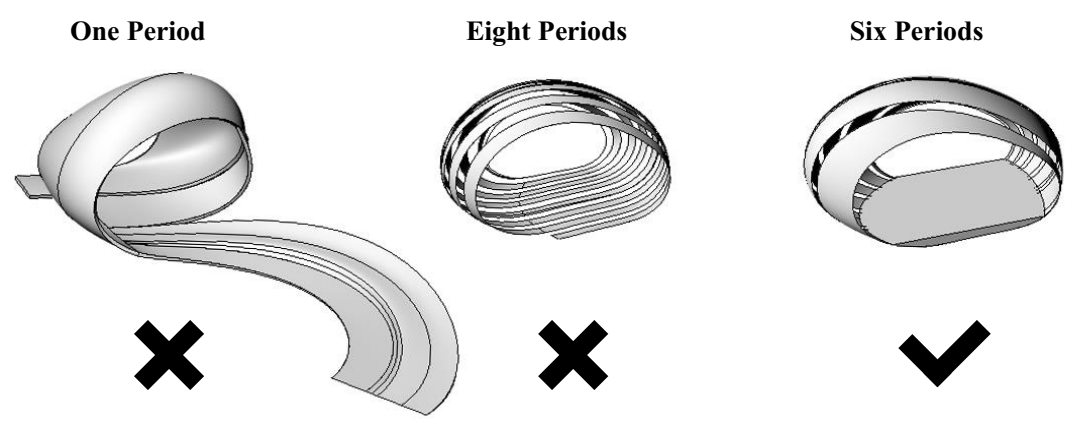

Figure 5. Wave with different frequencies generate different evaluation of architecture

\subsection{The schema generation of architecture functional logic}

The architecture form logic prototype is a schematization explanation of the wave. By translating the perceptual schema into the algorithm schema, period, frequency and amplitude have become the parameters that affect the generation of architecture mass. The original prototype of wave could not meet the functional requirement of the broadcasting studio. To make the architecture form more reasonable,we should make a topological transformation of the architecture prototype which is generated by the wave schema.

According to the requirements of design, the broadcasting studio should be divided into three main function areas: live broadcasting area, guided propagation area and leisure area. The live broadcasting area should be easy for lighting and have a good visibility. Moreover, it also could be divided into three areas respectively for radio host, guest and brief interactive performance. There should be a shoe changing area at the entrance of the studio. In addition, a small outdoor stage outside the studio is needed to make it convenient for the host to interact with passers-by when the program is on air. Then, we need to obtain a form logic from topological transformation of the wave schema to orgnize these functions rationally.

When we listen to the radio, a song, a word said by the host or even a story told by the audience may touch our hearts. The song may be a consolation when you are tired of the hard life; A story told by smoeone may strike a responsive chord among us. But there is nothing outside — not the broadcast content or the radio wave--touch our hearts. There is just the radio wave which is inside our hearts 
meet itself. In other words,we are just moved by ourselves. Accordingly, the schema of wave intersecting itself which is extracted from the perceptual image we depicted above formates a topological transformation structure of the architecture prototype. Extending the head and the tail of the wave and intersecting them inside the space so that it divides the space into two function areas---live broadcasting and leisure area,guided propagation area. Extending the head and the tail of the wave continusly in order to fomate the outdoor stage and LED wall outside the space. In this way,the functional logic of the architecture's form which is extracted from the prototype of wave that has been established.

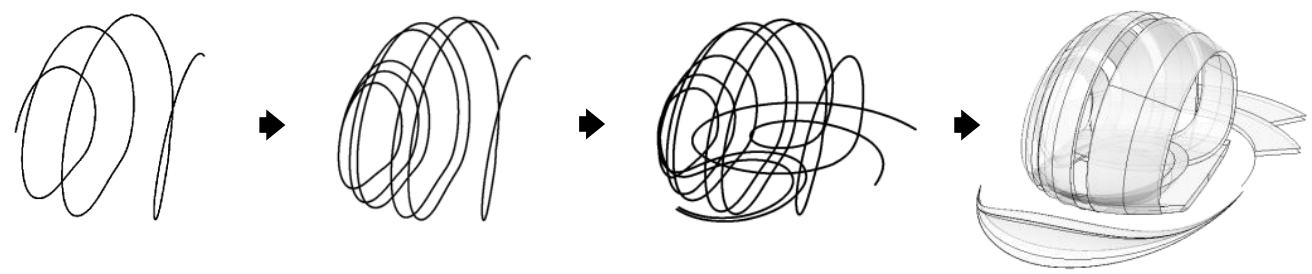

Figure 6. The generation of architecture form logic extraction from the schema of wave intersecting itself

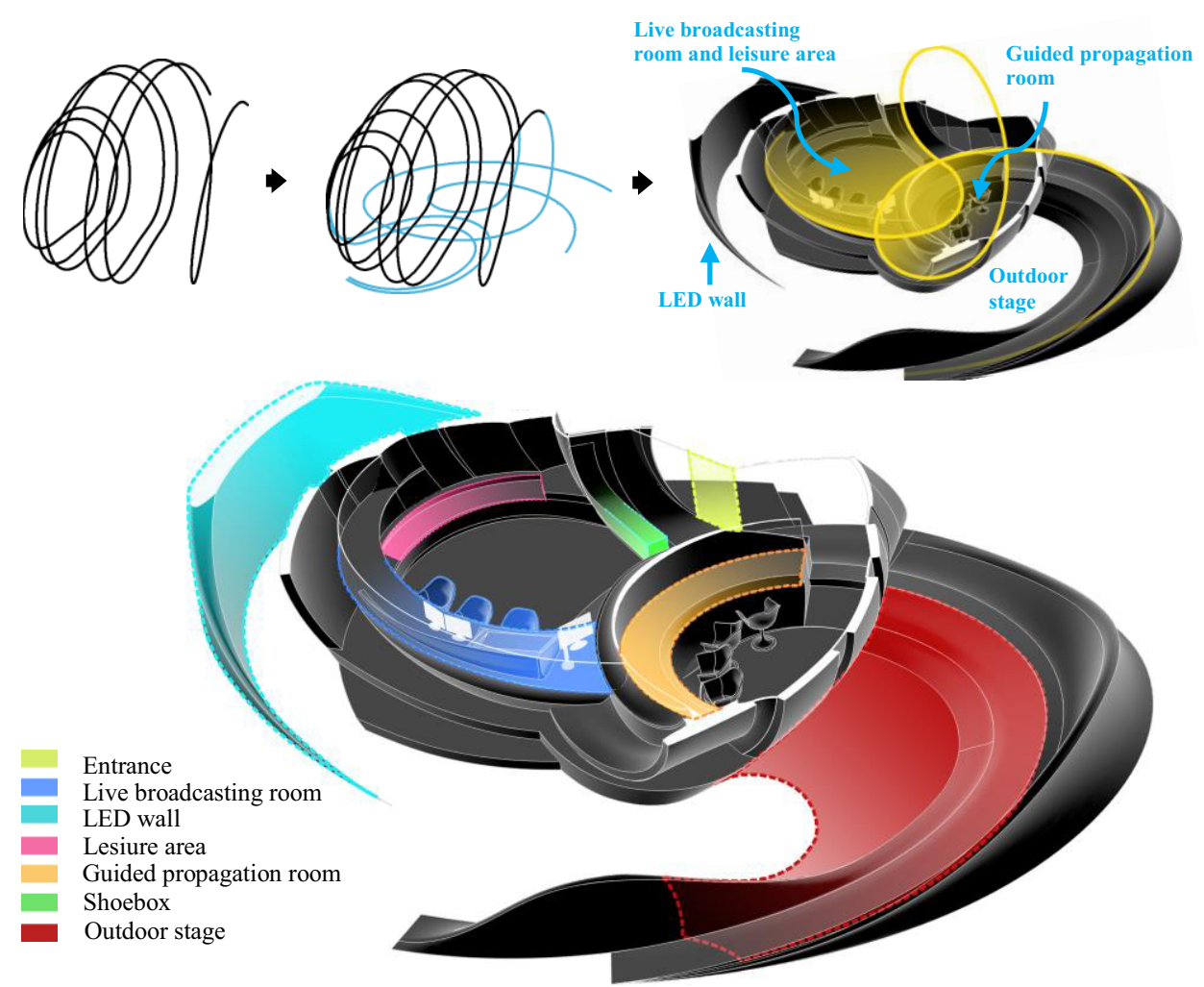

Figure 7. Functional divisions of architecture 


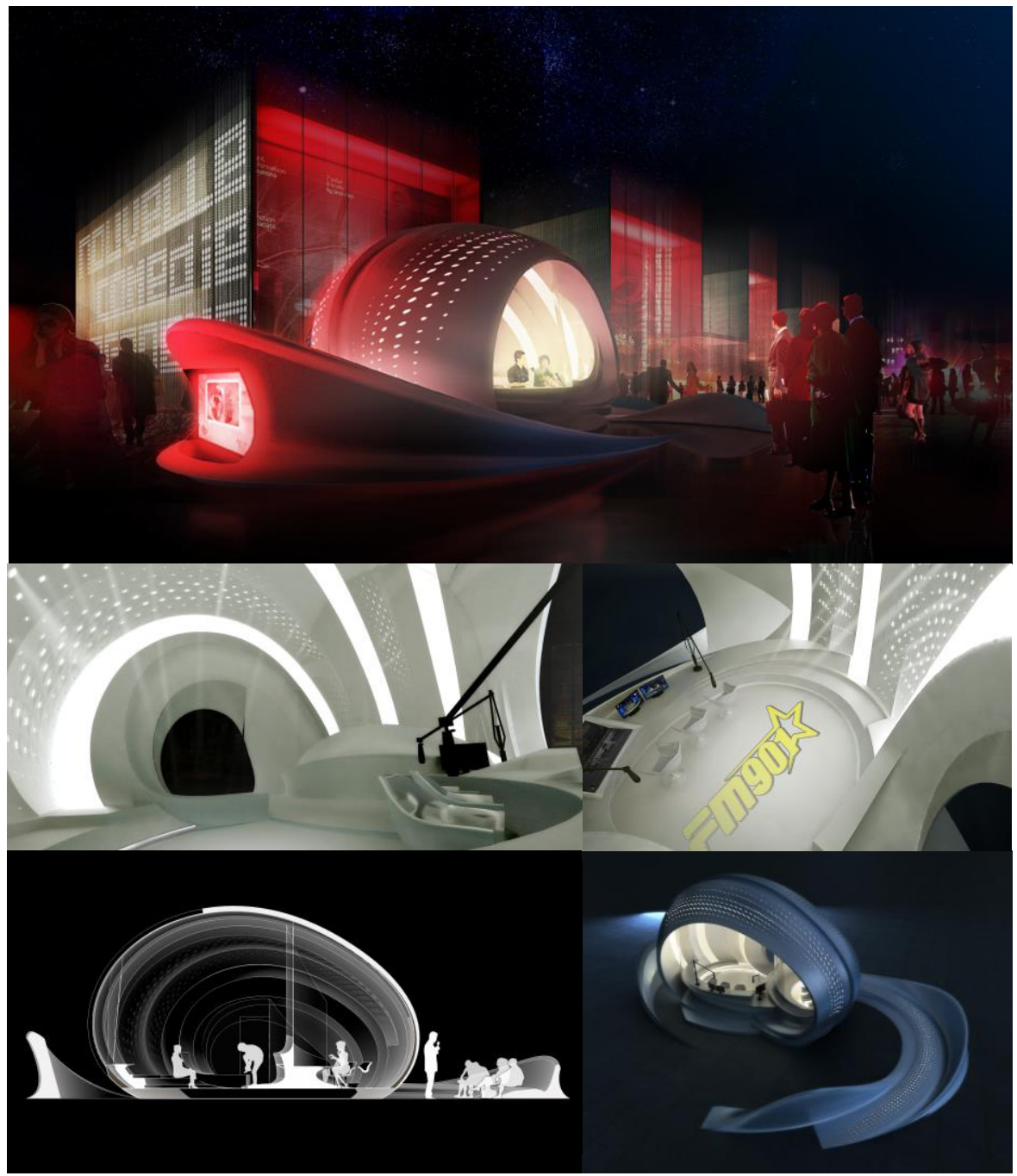

Figure 8. Rendering image of the outdoor broadcasting studio

\section{Conclusion}

Schema can help us cognize the architecture form logic generation. The abstract logic which has effects on form generation could be understood through the way of perceptual schema. In addition, the abstract schema structure can be projected to architecture form logic generation, therefore a scheme logic could be established to generate rational architecture form in the way of parametric design. This paper explores a new method to use the schema theory combining the parametric design through the case of outdoor broadcasting studio. The purpose of this paper is to investigate the relevance of scheme logic in scheme cognition and parametric design, so as to seek a new way of architecture design method under the new environment of architecture design.

\section{References}

1. J. Piaget, The Principles of Genetic Epistemology(Rout ledge and K. Paul, London, 1972) 
2. C.Alexander, A Pattern Language(Oxford University Press, London,1977)

3. Weiguo $\mathrm{Xu}$, Architectural Creation and Thinking of the Parametric Design in China: Interview with Professor Xu Weiguo \& Xu Feng of School of Architecture of Tsinghua University, Urban Environment Design, 35,108 (2009)

4. Tianyu Zhang, Yukun Zhang, Di Wang, Space Organization of Architecture and the Psychological Schemata, Huazhong Architecture, 24,65(2006)

5. Li Zhu, Nan Zhang, Xiaoying Qing, Research on Algorithm Schema of Parametric Architecture Design Based on Schema Theory, Advanced in Intelligent Systems Research,126,576(2015)

6. Xiong Wang, Guoping Pang,Schema Theory and Its Teaching Significance, Journal of Yulin Teachers College,25,98(2004) 